\title{
Low threshold frequency comb generation in AIGaAs-on-insulator microresonator in the normal dispersion regime
}

\author{
Kamel, Ayman Nassar; Pu, Minhao; Yvind, Kresten
}

Published in:

2017 Conference on Lasers and Electro-Optics

Link to article, DOI:

10.1364/CLEO_QELS.2017.FTu1D.3

Publication date:

2017

Document Version

Peer reviewed version

Link back to DTU Orbit

Citation (APA):

Kamel, A. N., Pu, M., \& Yvind, K. (2017). Low threshold frequency comb generation in AlGaAs-on-insulator microresonator in the normal dispersion regime. In 2017 Conference on Lasers and Electro-Optics Optical Society of America (OSA). https://doi.org/10.1364/CLEO QELS.2017.FTu1D.3

\section{General rights}

Copyright and moral rights for the publications made accessible in the public portal are retained by the authors and/or other copyright owners and it is a condition of accessing publications that users recognise and abide by the legal requirements associated with these rights.

- Users may download and print one copy of any publication from the public portal for the purpose of private study or research.

- You may not further distribute the material or use it for any profit-making activity or commercial gain

- You may freely distribute the URL identifying the publication in the public portal 


\title{
Low Threshold Frequency Comb Generation in AlGaAs-on- Insulator Microresonator in the Normal Dispersion Regime
}

\author{
Ayman Nassar Kamel, Minhao Pu and Kresten Yvind \\ DTU Fotonik, Technical University of Denmark, Building 343, DK-2800 Lyngby, Denmark \\ aynaka@fotonik.dtu.dk
}

\begin{abstract}
We present milli-Watt threshold frequency comb generation in AlGaAs-on-insulator integrated microresonators exhibiting normal GVD by employing the effects of mode interaction. OCIS codes: (190.4390) Nonlinear optics, integrated optics; (190.4970) Parametric oscillators and amplifiers.
\end{abstract}

\section{Introduction}

Optical frequency combs have advanced metrology and spectroscopy. The generation of frequency combs from integrated Kerr microresonators is very attractive for many applications, including optical communications [1]. The generation of frequency combs in such microresonators is based on parametric frequency conversion in four-wave mixing processes. Efficient generation of frequency combs in those systems requires a high Kerr nonlinearity and tight confinement of light, as well as phase matching. In most demonstrations of Kerr frequency comb generation, microresonators are designed to achieve anomalous group velocity dispersion (GVD) at the pump wavelength to satisfy the phase matching condition and generate coherent combs via soliton generation [2]. However, there is a growing interest in generating frequency combs in the normal dispersion regime. This is beneficial when it is difficult to achieve anomalous dispersion or when the requirements to achieve anomalous dispersion conflict with other important merits such as the quality factor. In addition, coherent frequency comb generation has been theoretically predicted and experimentally observed in the normal dispersion regime when the dispersion is locally disturbed by mode interaction [3, 4]. The AlGaAs-on-insulator platform offers a very high Kerr nonlinearity. In addition, due to its high index contrast between the core and the cladding materials, it also offers tight confinement of light. Those two features have allowed the generation of frequency combs with very low threshold powers [5]. However, the high index contrast also results in bigger scattering loss due to the sidewall roughness. To mitigate this effect, we design microresonators with a wider cross-section so that the overlap of the fundamental mode profile with the sidewalls is decreased. The wider microresonators exhibit normal GVD. The microresonator supports a higher-order mode that interacts with the fundamental mode, shifting the modes frequencies, which makes phase matching possible. Since the two mode families have different FSRs, they interact periodically giving rise to a periodic shift in the dispersion and resonances for which the phase matching condition is satisfied.

\section{Dispersion disturbance by mode interaction}

The microresonator, made from $\mathrm{Al}_{0.17} \mathrm{Ga}_{0.83} \mathrm{As}$, has a thickness of 270nm, a width of 900nm and a perimeter of $810 \mu \mathrm{m}$. The bus waveguide is integrated on-chip and, together with the microresonator, are embedded in $\mathrm{SiO}_{2}[6]$. The fundamental mode FSR is $102 \mathrm{GHz}$ and the higher-order mode FSR is $89 \mathrm{GHz}$ at $1550 \mathrm{~nm}$, which results in an interaction period of around $6 \mathrm{~nm}$. The dispersion of the fundamental mode is characterized by measuring its FSR in a wide wavelength range. The dispersion measurment setup is shown in Fig.1.(a). An external-cavity diode laser (ECDL) is coupled to the waveguide by a lensed fiber and the ouput light is collected by another lensed fiber. The laser frequency is swept and the transmitted light is detected by a photodiode (PD). Simultaneously, a part of the laser light passes through a free-space Mach-Zehnder interferometer (MZI) which has a FSR of 2490MHz and its output is detected by another PD. The PD signals are recorded by an oscilloscope. The MZI is used to accurately calibrate the laser frequency shift while the scan is running. Fig.1(b) shows the measured FSR of the fundamental mode. The FSR increases with wavelength giving normal GVD and exhibits disturbances of up to a few GHz.
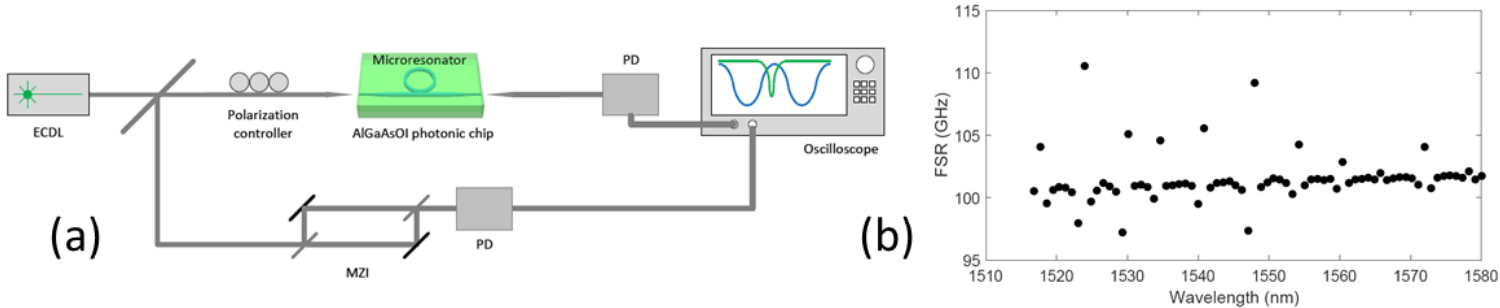

Fig.1 (a): Dispersion measurement setup, (b) measurement of the FSR of the fundamental mode 


\section{Comb generation}

Mode interaction results in blue (red) shifted modes, which appears as a decrease (increase) in the FSR. Because of this disturbance of the dispersion, there are sets of modes for which the FSR is effectively decreasing with wavelength, satisfying the phase matching condition. To demonstrate comb generation in our device, we use the setup shown in Fig.2 (a). Light from an ECDL is amplified by an Erbium-doped fiber amplifier (EDFA) and is coupled to the chip by a lensed fiber. The output light is collected by another lensed fiber and is detected by an optical spectrum analyzer (OSA). The input laser is tuned on resonance from the blue side of a resonance with a loaded $Q$ of 190,000 and $9 \mathrm{~dB}$ extinction around 1548nm. Fig.2 (b) shows the output spectrum at the threshold power of $7 \mathrm{~mW}$. When the input power is increased to $30 \mathrm{~mW}$, the spectrum shown in Fig.2 (c) results. This spectrum has dominant peaks separated by around $6 \mathrm{~nm}$, which is equal to the interaction period of the mode families. This suggests that phase matching results because of mode interaction.

(a)

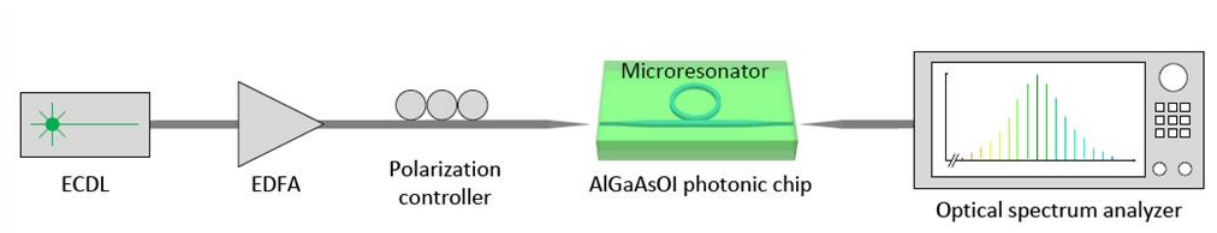

(b)

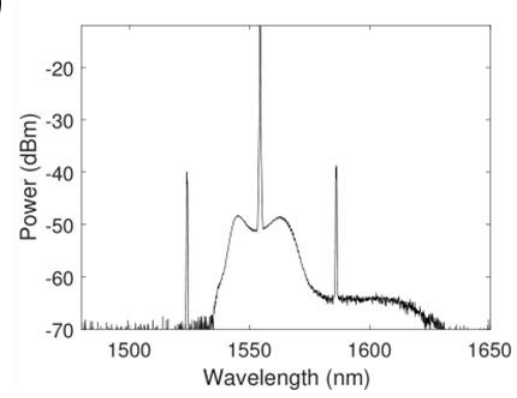

(c)

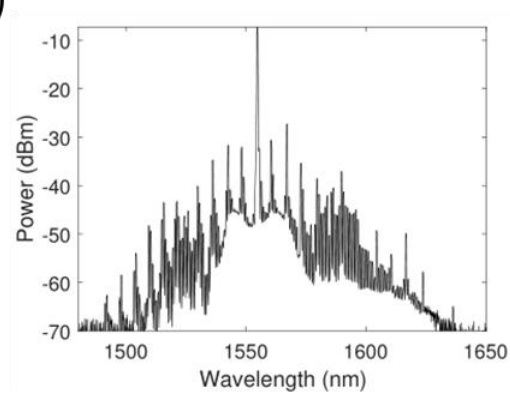

Fig.2 (a): The setup employed for frequency comb generation, (b) the obtained spectrum at the threshold power of $7 \mathrm{~mW}$, (c) the obtained spectrum at a power of $30 \mathrm{~mW}$

\section{Conclusion}

We have demonstrated frequency comb generation in an AlGaAs-on-insulator microresonator with normal dispersion through mode interaction. This regime of comb generation allows the use of wide microresonators that exhibit higher quality factors, resulting in lower threshold power.

\section{Acknowledgements}

The authors acknowledge financial support from the Danish Research Council and Villum Fonden via the SPOC (DNRF123), and NATEC centers of excellence.

\section{References}

[1] Kippenberg, T. J., Holzwarth, R.., Diddams, S. A., "Microresonator-based optical frequency combs.," Science 332(6029), 555-559 (2011).

[2] Herr, T., Brasch, V., Jost, J. D., Wang, C. Y., Kondratiev, N. M., Gorodetsky, M. L.., Kippenberg, T. J., "Temporal solitons in optical microresonators," Nat. Photonics 8(2), 145-152, Nature Publishing Group (2013).

[3] Lobanov, V. E., Lihachev, G., Kippenberg, T. J.., Gorodetsky, M. L., "Frequency combs and platicons in optical microresonators with normal GVD," Opt. Express 23(6), 7713-7721 (2015).

[4] Xue, X., Xuan, Y., Liu, Y., Wang, P.-H., Chen, S., Wang, J., Leaird, D. E., Qi, M.., Weiner, A. M., “Mode-locked dark pulse Kerr combs in normal-dispersion microresonators," Nat. Photonics 9(9), 594-600, Nature Publishing Group (2015).

[5] Pu, M., Ottaviano, L., Semenova, E.., Yvind, K., "Efficient frequency comb generation in AlGaAs-on-insulator," Optica 3(8), 823-826 (2016).

[6] Ottaviano, L., Minhao, P., Semenova, E.., Yvind, K., "Low-loss high-confinement waveguides and microring resonators in AlGaAs-oninsulator," Opt. Lett. 41(17), 3996-3999 (2016). 